that condensations of stellar magnitude would be formed in the arms (the spiral form of 'which, however, he could not account for) ; that some of these might eventually break up by fission into close double stars, but that wide doubles were probably formed by a capture process; that nothing resembling a solar system could be produced by rotation alone, and that the hypothesis of Kant and Laplace in at least its original form was untenable; that, as an alternative, the hypothesis of disruption by a tidal encounter fitted many of the facts; but that if this were the actual origin of the solar system, then planetary systems similar to it must be very rare in Nature, so rare that possibly ours is the only one.

It is the usual fate of cosmogonic theories not to survive, and many of Jeans' conclusions, especially since the discovery of the expansion of the universe, are already in course of revision. But "Problems of Cosmogony" was grand stuff from beginning to end. We saw a master thinker and executant at work with his materials. He set a standard of perfection of scholarship in a field often marred in others' hands by propaganda; the work was marked by power, depth and originality of a high order.

His later volume, "Astronomy and Cosmogony", more comprehensive and more ambitious, was less successful. Besides reconsidering and enlarging much of the Adams Prize essay, it aimed at giving an account of the internal constitution of the stars on rather different lines from the work of Eddington. Some of Jeans' mathematics in this volume is far from clear. His conclusions that the different types of stars-giants, main sequence stars and white dwarfs-correspond to the successive removals of the $M, L$ and $K$ rings of electrons from atoms by ionization are hard to accept. But his other conclusions, that the stars in general have 'liquid', not gaseous, cores, and consist of elements of atomic number 95 or so, are perhaps more acceptable to-day than when they were published. There is, however, scarcely a page of even this second treatise which is not rich in fascinating suggestion and inspiring possibility; on each page we see a master mind confronting itself with the grandest problems, of formidable difficulty, posing them, simplifying them, and making some progress with even the most intractable.

Jeans influenced astronomical investigation in many other domains besides those mentioned above. He first gave the name 'equation of transfer' to the equation which traces the intensity of a pencil of radiation through an absorbing and emitting medium, an equation in daily use in investigations on stellar atmospheres; and he first directed attention to the phenomenon of radiative viscosity...He made the suggestion that the source of stellar energy might be the mutual annihilation of protons and electrons; and he, more than anyone, stood for the belief that the universe is doomed to a 'heat-death'. He did not form a school of research in the ordinary sense; but everyone who is interested in the beginnings, evolutions and endings of the various members of our universe is in a sense his pupil. We have lost a great leader.

Jeans had a brisk business-like manner in ordinary conversation, not at all suggesting the deep academic thinker that he was; but it developed in the lecture theatre into a winning persuasiveness. He was extremely modest, a most courteous correspondent and a scrupulously fair opponent in a controversy; and he gave freely of his friendship to many. $\mathrm{He}$ was twice married.
E. A. MiLne

\section{Prof. Otto Hönigschmid 26/6}

By his untimely death during October 1945, a tragic victim of trar cofitions, chemical science has lost in Prpf. Pttp. Nontschmid one of its leading workers in fiell f $f$ inorganic chemistry. His name will alwall be remehbered in chemical circles as the outst pndng authority in Europe on the chemical ator weights, and for the active school of research fi dilt up at Munich after his appointment there in 12 as professor ordinarius.

Born in Horovitz in Bohemia in 1878, he graduated in the German University of Prague and studied as a research student under Moissan in Paris (1909-10) and later with Th. W. Richards at Cambridge, Mass., where he learnt and applied the methods developed at Harvard to the determination of the atomic weight of calcium.

At that period, cumulative evidence from various sources, notably from the atomic weight laboratory at Harvard and from Guye's physical chemistry school at Geneva, had shown that Stas' classical values for the fundamental atomic weight ratios were affected by significant and hitherto unsuspected errors. Stas' value for silver, the standard to which all his other values were referred, came under suspicion, and his value for nitrogen had been shown by Guye and others to be too great by as much as one part in four hundred. Since at that time the measurement of stoichiometric ratios afforded the only means of finding atomic weights with accuracy, density methods being regarded as approximate only, a general revision became imperative. This had already been made for many of the common elements by Richards and his co-workers, who had developed to a high degree the very exacting preparative and analytical technique essential in this field of work. Much, however, remained to be done, and Hönigschmid on his return to Europe dedicated his energies to researches of this nature.

In Vienna, Hönigschmid made, at this period, what was then claimed to be the first really accurate determination of the atomic weight of radium, using as the starting material preparations containing 840 mgm. of the pure element. Twenty-two years later, this work was repeated in Munich, using the much larger quantity of $3 \mathrm{gm}$. of element, put at his disposal by the Union Minière du Haut Katanga, of Brussels, and which was initially 98.83 per cent pure. This large quantity enabled a very effective fractional crystallization to be made without reducing unduly the quantity of the final pure material. The atomic weight found was $226 \cdot 05$, the present International value; which was only 0.08 unit greater than the earlier one.

Hönigschmid's most active period of research began after his promotion from the directorship of the inorganic and analytical laboratories at the Technische Hochschule, Prague, to the University of Munich, where he founded a laboratory devoted entirely to the determination of atomic weights by analytical methods.

From this laboratory, year after year, appeared a stream of papers embodying improved techniques for the preparation of chemically pure substances, and for their analysis, and containing fresh data of the highest accuracy on atomic weight ratios. Up to 1938 he and his co-workers had re-determined the atomic weights of upwards of forty elements, which led to numerous revisions in accepted values.

Hönigschmid was the first to determine the atomic 
weights of hafnium and rhenium, and his careful work on radium, thorium, uranium and ionium as well as on the radiogenic leads contributed in no small degree to the body of direct chemical evidence which supports Rutherford and Soddy's theory of radioactive disintegration.

To the Munich school belongs the chief credit of establishing on a firm basis that important substandard, the atomic weight of silver, with sufficient accuracy to enable it to be used as a reference standard for the halides of elements of low atomic weight such as lithium and sodium. Hönigschmid directed attention to the small difference between the atomic weight of silver derived from the synthesis of silver nitrate made in 1907 by Richards and Forbes and that from the lithium perchlorate/lithium chloride/ silver ratios measured three years later by Richards and Willard. The former, assuming nitrogen to be $14 \cdot 008$, gave for silver $107 \cdot 879$, whereas the latter yielded $107 \cdot 87 \mathrm{l}-\mathrm{a}$ small difference indeed, but one which would cause a much greater uncertainty in the atomic values for light elements. To investigate this, Hönigschmid, Zintl and Thilo re-determined the nitrate ratios by the reduction in hydrogen of specially purified silver nitrate, and by this analysis obtained precisely the same value, to 1 part in 150,000 , for the ratio silver nitrate/silver as that found by synthesis eighteen years earlier at Harvard.

This research, published in 1927, was followed in 1929 by the work of Hönigschmid and Sachtleben in which barium perchlorate was used to link silver to oxygen. These two researches led to closely concordant results which confirmed the higher of the two values for silver and went far to settle the discrepancy. Subsequent work on silver has supported Hönigschmid's conclusion, and the uncertainty now in this standard probably does not exceed two units in the third place of decimals.

The development of the mass spectrograph by Aston, and the discovery that many chemical elements were mixtures, gave a new direction and impetus to chemical work. Chemists were concerned to know how much reliance could be placed on the new physical method and whether the very significant differences between many of the chemical and physical values were to be explained by chemical or by physical errors. On both sides of the Atlantic, in the two chief schools for this type of work, at Harvard and at Munich, numerous re-determinations were undertaken, with the result that in most cases the mass spectrograph values were found the more reliable and pointed the way to the elimination of chemical errors. In these activities Hönigschmid and his pupils played an important part. For example, his work on niobium and tantalum is outstanding, as also is his work on the atomic weight of phosphorus. In all three cases he proved that the accepted chemical values were markedly too high, and that when really pure halides, or oxyhalide in the case of phosphorus, were used a close concordance with the mass spectrograph was obtained.

In a few instances, however, errors were discovered in the mass spectrograph values, and chemical revision even with extreme elaboration failed to bring the two sets of data into accord. Such was the case with cadmium and tellurium, which when revised chemically by Hönigschmid paved the way to the discovery of new isotopes of tellurium and a modification of the abundance ratios for cadmium. Another example is that of neodymium, the chemical value of which when revised by Hönigschmid and Wittner was found in agreement with the earlier value of Baxter and Chapin, and nearly 0.8 unit higher than Aston's value, which has now been shown by Mattauch to have been based on an isotopic constitution affected by small errors.

Mention should be made, too, of Hönigschmid's application of precise analytical methods to detect and measure the degree of isotopic separation achieved by evaporation at low pressure, in the pioneer experiments of Brönsted and Hevesy on mercury and on lead chloride and by Hevesy alone on potassium. In those for mercury and potassium the separation was small but definitely detectable. Much later, he tested the isotopic hydrogen chlorides obtained by Clusius and Dickel in their thermal diffusion apparatus, and confirmed beyond doubt that the separation was practically 100 per cent.

Dúring the earlier part of the War, Hönigschmid appears to have continued his atomic weight work for, in 1941, he published papers on zinc, samarium, and yttrium. Later work from his laboratory has not been reported.

Finally, the services rendered to the German Atomic Weight Commission by Hönigschmid, who was chairman during 1920-30, must be noted. The eleven yearly reports and reviews of the progress of researeh with the annual table of the most trustworthy atomic weight values came mainly from his pen. In 1930, when the Atomic Weight Committee of the International Union of Chemistry came into being, Hönigschmid was the obvious choice for the German representative. Under the chairmanship of Prof. G. P. Baxter, eleven international reports have been issued since then, and although from time to time there has been a change in membership, Otto Hönigschmid and his opposite number, G. P. Baxter, the leaders of the two chief schools of atomic weight research in Europe and America, have invariably been re-elected.

Enough has been said in this notice to indicate the great part played by Otto Hönigschmid in analytical and atomic weight research. It is a matter of general regret that work like his, of a truly international character, should have been abruptly terminated before his energies kere exhausted.

\section{R. WhytLAW-GRAY}

\section{Prof. I. Mościcki}

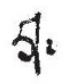

Prof. Ignacy Mościcki, former President of Poland and a lytinguished chemist, died on October 2 at Versoix, foneva, in his seventy-ninth year.

Borm at Mierzanow, then in Russia, Mościcki was educated locally and at Riga, but his revolutionary viou mecessitated his leaving Russia. For five years he stayed in England, spending some time at Finsbury Technical College. Then he went to the University of Fribourg as assistant to Prof. Kuwalski, under whose influence he became interested in the applica. tions of science and made a careful study of the methods for the fixation of nitrogen. He invented new processes that proved successful, and in 1913 he was appointed professor of electrochemistry at Lwów (then in Austria).

After the liberation of Poland, Mościcki became head of the chemical works at.Chorzów and Mościce (named in his honour). He succeeded in establishing the Polish chemical industry on a firm basis, and 